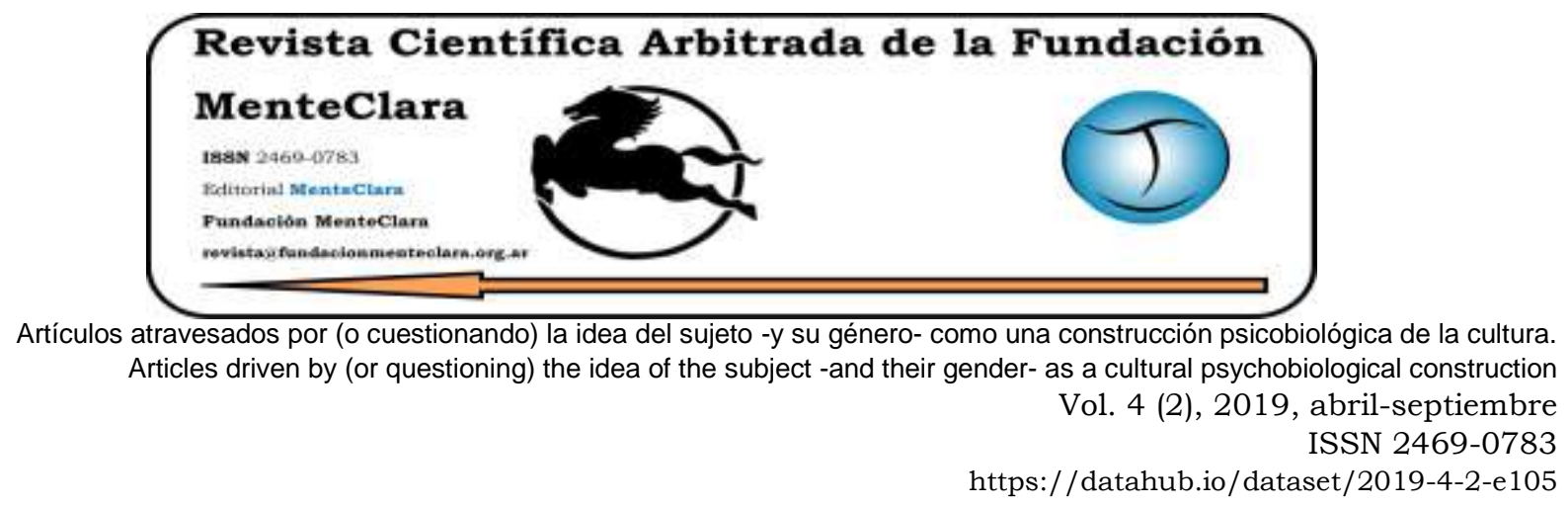

\title{
MODELOS SOCIOPOLÍTICOS EN DISPUTA. EL CASO DEL BLOQUEO A HUAWEI
}

\author{
SOCIOPOLITICAL MODELS IN DISPUTE. THE CASE OF HUAWEI'S COMMERCIAL \\ BAN
}

Juan Pablo López Alurralde ${ }^{1}$ Gastón Becerra²

Cómo citar este artículo / Citation: López Alurralde, J.P. \& Becerra, G. (2019). "Modelos sociopoliticos en disputa. El caso del bloqueo a Huawei". Revista Científica Arbitrada de la Fundación MenteClara, 4(2) abril-septiembre 2019, 155-163. DOI: https://doi.org/10.32351/rca.v4.2.105

Copyright: (C) 2019 RCAFMC. Este artículo de acceso abierto es distribuido bajo los términos de la licencia Creative Commons Attribution 4.0 International License (CC BY 4.0). Recibido: 30/09/2019. Aceptado: 05/10/2019 Publicación online: 30/10/2019

Conflicto de intereses: Ninguno que declarar.

\section{Resumen}

No puede establecerse con claridad de qué manera el desarrollo digital moldeará las relaciones económicas y sociales del mundo interconectado, sobre todo en vistas a la inminente revolución aparejada a la aparición de redes $5 \mathrm{G}$ e internet de las cosas. Lo que está claro, es que los valores y pilares bajo los que se fundan se encuentran en abierta discusión y disputa.

En esta breve comunicación, tomando como punto de partida el actual enfrentamiento comercial y tecnológico entre Estados Unidos y Huawei, se tratará de identificar y caracterizar dos modelos sociopoliticos enfrentados que resuelven a su manera el eje de relaciones entre individuos, gobiernos y compañias. En particular, se ahondará en el reconocimiento de dos escenarios diferentes. Por un lado, Occidente, donde pueden encontrarse a grandes compañias como Google y Facebook siendo interpeladas por actores del ámbito civil, judicial y politico, y donde pesan

\footnotetext{
${ }^{1}$ Universidad Nacional de Quilmes, Argentina. juanlopezalurralde@gmail.com

2 Universidad de Buenos Aires - Universidad Abierta Interamericana, Argentina. gaston.becerra@sociales.uba.ar
} 
importantes acusaciones de competencia desleal y manipulación de datos privados; y, por otro lado, China, donde las autoridades "protegen" a sus 800 millones de internautas para la explotación de unas pocas compañias en lo que puede entenderse como un peligroso modelo de integración vertical.

\begin{abstract}
It cannot be clearly established how digital development will shape the economic and social relations of the interconnected world, especially in view of the imminent revolution coupled with the emergence of $5 \mathrm{G}$ networks and IoT. What is clear is that the values and pillars under which they are grounded are in open discussion and dispute. In this brief communication, taking the current commercial and technological confrontation between the United States and Huawei as a starting point, we will try to identify two confronted sociopolitical models that resolve in their own way the axis of relationships between individuals, governments and tech companies. In particular, it will delve into the recognition of two different scenarios. On the one hand, the West, where large companies such as Google and Facebook are systematically questioned by civil, judicial and political spheres, while they deal with important accusations of unfair competition and manipulation of private data; and, on the other hand, China, where the authorities "protect" their 800 million Internet users for the exploitation of a few companies in what can be understood as a dangerous model of vertical integration.
\end{abstract}

Palabras Claves: innovación digital; modelos sociopolítico; internet de las cosas

Keywords: digital innovation; sociopolitical models; interenet of things 


\section{Introducción}

Vivimos en una actualidad en la que lo informacional y lo tecnológico están profundamente entrelazados con lo social, lo político, lo económico y hasta lo ecológico (Latour, 1999). Ejemplos de ello son la emergencia del big data, especialmente a partir de la interacción que proponen plataformas sociales en las cuales, de forma voluntaria, los usuarios dejan registro de sus intereses y de sus consumos compartidos con otros usuarios, a sabiendas -o no tanto- que dicha información constituye un medio para la publicidad y la propaganda (Byung-Chul, 2014), (Ritzer, 2014). O por caso, la tecnología 5G, lejos de tratarse de una mera innovación tecnológica, es vista por muchos analistas como un milestone para el próximo salto en el desarrollo de la internet de las cosas (Sequeira, 2019), y por esta vía, una profundización del proceso de automatización en distintos sectores, lo que podria desencadenar otra crisis por falta de empleo (Rifkin, 1996).

Dado este escenario, resulta sorprendente la emergencia de un discurso que parece negar tanto el basamento como el impacto físico y social de las nuevas tecnologías. Uno de estos discursos, por ejemplo, es el que rodea al concepto de "infoesfera" propuesto por el filósofo Luciano Floridi:

"Minimamente, la infosfera denota todo el entorno informativo constituido por todas las entidades informativas, sus propiedades, interacciones, procesos y relaciones mutuas. Es un entorno comparable pero diferente del ciberespacio, que es solo una de sus subregiones, por asi decirlo, ya que la infosfera también incluye espacios de información fuera de línea y análogos. Máximamente, la infosfera es un concepto que también puede usarse como sinónimo de realidad, una vez que interpretamos este último de manera informativa. En este caso, la sugerencia es que lo que es real es informativo y lo que es informativo es real. Es en esta equivalencia que se encuentra la fuente de algunas de las transformaciones más profundas y problemas desafiantes que experimentaremos en el futuro cercano, en lo que respecta a la tecnología" (Floridi, 2002, p. 41). 
Esta materialidad -social y física- que se esconde en el mundo de la información se descubre en la energía y los recursos que necesitan su infraestructura, en los usos habilitados y en los límites de las regulaciones (Portmess \& Towers, 2015).

En esta breve comunicación nos interesa hacer foco en un caso reciente para ilustrar la tensión entre dos modelos que plantean de manera particular las relaciones entre individuos, empresas y gobierno.

\section{El caso del bloqueo a Huawei}

Entre el 19 de mayo y el 29 de junio de 2019 la empresa Huawei fue incluida en una entity list por parte del gobierno de Donald Trump, a partir de la cual no podría hacer negocios con empresas norteamericanas. Entre las razones esgrimidas para justificar dicho bloqueo se encuentra, en primer lugar, el riesgo de sufrir espionaje industrial y el robo de secretos tecnológicos. De acuerdo con los periodistas Ana Swanson y Paul Mozur (2019) del New York Times, Trump “... hace lucir cada vez más borrosa la linea que hay entre la seguridad nacional y la seguridad económica de Estados Unidos, lo que le permite aprovechar poderosas herramientas diseñadas para castigar a los peores actores globales del mundo y redirigirlas hacia casi cualquier socio comercial".

Huawei, reconocida principalmente por sus marcas de teléfonos celulares que han crecido exponencialmente acaparando una cuota importante del mercado de telefonía móvil, ofrece también una gama de servicios de conexión a internet, donde cobran vital importancia las infraestructuras relativas a la conectividad 5G. Con el desarrollo de esta última tecnología, los gobiernos y analistas auguran y pronostican una profundización de la digitalización. Mientras las conectividades 3G y 4G renovaron las funcionalidades y experiencias de la conectividad móvil de 
los usuarios, se espera que la conectividad 5G implique una revolución en las comunicaciones máquina a máquina. Si bien el incremento de velocidad que comporta es notable, el mismo no debería influir en la experiencia normal de los usuarios. En cambio, lo que marca una distinción importante es la reducción de la latencia en la comunicación entre dispositivos. Con este tipo de conectividad se espera que sea posible, por ejemplo, vincular todos los automóviles de una ciudad a un mismo sistema de conducción autónoma, y así delegar y automatizar varios procesos como el transporte de elementos.

Lo que subyace al conflicto es el enfrentamiento de dos modelos tecnológicos diferentes, con dos modos de entender el desarrollo y la apropiación de la tecnología. O, en otras palabras, dos formas de resolver la relación entre usuarios -individuos-, servicios -compañias- y gobiernos -eje de relaciones que determinará las posibilidades de desarrollo e incorporación tecnológica como sus subsecuentes facultades de dinamización económica-. Por ello cabe preguntarse: ¿Cuáles son los escenarios, los perfiles y las agendas de políticas digitales de cada lado del mapa? ¿Qué tipo de regulaciones y libertades se confieren en cada caso al desarrollo tecnológico y qué razones aportan estas para justificar medidas como la prohibición a Huawei? ¿Qué aspiraciones tienen puesta en el desarrollo digital y cuáles son las demandas e intereses de los usuarios e individuos de la sociedad civil?

En lo que respecta a Europa y Estados Unidos, los últimos años nos han mostrado una serie de fuertes estridencias entre gobiernos $\mathrm{y}$ compañias digitales cuyo conflicto termina, por un lado, con una agenda política cargada de normativas y discusiones regulatorias, y con la creciente necesidad de las compañias de defender su inimputabilidad. Tales sucesos pueden verse claramente ilustrados en la sanción de la normativa GPDR, que regula el acceso y manipulación de datos 
personales en el ciberespacio, y en la imagen, más icónica todavía, de Mark Zuckerberg defendiéndose en los estrados de la Cámara de Senadores de EEUU a principios del año pasado. Esta imagen, que nos muestra a las firmas más ricas del planeta siendo públicamente cuestionadas por sus gobiernos y audiencias, debe ser entendida como una consecuencia del escándalo de Cambridge Analytica, que puso al descubierto su capacidad para constituirse como un actor central en el proceso político, aun cuando su impacto real en los rumbos electorales se pueda poner seriamente en duda (Galup, 2019). Y si bien uno podría pensar que dicha imagen no es más que un intento de expiación de la clase política, eso no invalida el mensaje público de que es necesario estar alerta de lo que hacen un puñado de firmas con el conocimiento de lo que miles de millones de usuarios hacen a diario.

¿Qué pasa en China? Desde hace años, el gobierno ha establecido un cerco a todas las firmas digitales extranjeras que representan algún grado de amenaza para sus compañias locales. ¿Google en China? Negativo ¿Amazon o Facebook? Tampoco. En su lugar, y en lo que puede entenderse como una suerte de proteccionismo digital, China ha mantenido su vasto ciberespacio fuera del alcance de Silicon Valley. Sin embargo, hay algo más preocupante a los ojos occidentales: en el año 2014, el Consejo de Estado aprobó una iniciativa que busca terminar para el año 2020 un sistema de seguimiento de individuos e instituciones conformado por la convergencia de bases de datos tanto públicas como privadas. Semejante esquema de triangulaciones puede entenderse bajo la noción de integración vertical: las compañías ofrecen servicios y productos a sus usuarios que a su vez son explotados en beneficio de los intereses empresariales y estatales, generando una pirámide cuya base contiene a 800 millones de internautas y en su cúspide a unas pocas corporaciones bajo el ala protectora del estado y el partido. Esta 
capacidad de integración produce resquemores a la luz de las capacidades epistémicas que comporta el panoptismo digital. Y mucho más todavía cuando nos percatamos que en China los servicios digitales están soportados por compañias que, para ponerlo en términos familiares, amalgaman las funciones de Google, Amazon y Facebook.

\section{Conclusión}

El caso descrito nos devuelve un escenario de tensiones encontradas entre lógicas de producción y regulación, innovación y resguardo, competencia y protección. ¿Cómo se resuelve la tensión entre el resguardo de valores humanistas o democráticos, por un lado, y el interés por consolidar la supremacía tecnológica de sus firmas, frente a la competencia que impone un desarrollo tecnológico sin resistencias?

No podemos establecer con seguridad qué nuevas postales nos depare el futuro digital, aunque el mismo se encuentre sólo a 5 años. Por lo pronto, una "arqueología digital" nos demuestra algo inusual respecto al modo en que esta tecnología afecta nuestra vida. A diferencia de otras, no sólo se destaca su ratio de innovación incremental, sino, también, que repliegue sobre sí misma nuevas y nuevas capas de evolución digital. Al compás de esta convicción, gurúes y analistas profieren diagnósticos dispares: atrás quedaron las maravillas de las redes sociales y telefonía móvil. Hoy las compañias tienen las miradas puestas en inteligencia artificial e internet de las cosas. Piensan en un mundo donde todo un sector de la economía, el transporte, puede ser suplantado por algoritmos de conducción autónoma, y donde todas las tareas medianamente repetitivas y ordinarias pueden ser algoritmicamente automatizadas. Los enfoques optimistas auguran un crecimiento exponencial de las economías gracias a la tracción de la destrucción creativa. Desde la otra tribuna, lejos de querer parecerse a los ludistas del siglo XIX, inciden en 
la excepcionalidad de la revolución digital por venir: las máquinas reemplazan nuestra fuerza física en los circuitos de trabajo, y poco falta para que los algoritmos ocupen roles destinados a seres humanos en las relaciones sociales. El escenario es tan disruptivo que hasta los conservadores comienzan a plantear la necesidad de salarios universales para las masas de humanos posiblemente desplazados...

Con todo, el futuro digital es incierto. Mientras tanto, podemos suponer, en China y Occidente, será diferente. 


\section{Referencias}

Byung-Chul, H. (2014). Psicopolitica. Neoliberalismo y nuevas técnicas de poder. Barcelona: Herder.

Floridi, L. (2002). The 4th. Revolution. New York: Oxford University Press.

Galup, L. (2019). Big data y politica. Buenos Aires: Ediciones B.

Latour, B. (1999). Pandora's hope. Essays on the reality of science studies. Massachusetts: Harvard University Press.

Portmess, L., \& Tower, S. (2015). Data barns, ambient intelligence and cloud computing: the tacit epistemology and linguistic representation of Big Data. Ethics and Information Technology, 17(1), 1-9. https://doi.org/10.1007/s10676-014-9357-2

Rifkin, J. (1996). El fin del trabajo. https://doi.org/10.26754/ojs_ais/ais.19976131

Ritzer, G. (2014). Prosumption: Evolution, revolution, or eternal return of the same? Journal of Consumer Culture, 14(1), 3-24.

Sequeira, N. (2019). What 5G means for the future of the Internet of Things. ECN Magazine. https://www.ecnmag.com/blog/2019/01/what-5g-means-future-internet-things

Swanson, A., Mozur, P. (2019). Trump fusiona sus amenazas económicas y de seguridad nacional para presionar a aliados. The New York Times (ES).

https://www.nytimes.com/es/2019/06/10/aranceles-trump-seguridad-nacional 